\title{
Research on traffic data-collectiong system based on mc9s12d64 microcontroller
}

\author{
Jian Chen $^{1}$ \\ ${ }^{1}$ Shenzhen Polytechnic, Shenzhen 518055, China \\ akingders2@szpt.edu.cn
}

\begin{abstract}
Keywords: Intelligent Transportation System; Traffic data-collecting; System structure; CAN
\end{abstract} field-bus; Software design

Abstract. Traffic data-collecting is one of the basal contents of Intelligent Transportation System (ITS). Supply in the paper a design scheme of data-collecting system based on 9S12D64 microcontroller, furthermore, discuss in detail the system's operating principle, hardware structure and software design. This system has been developed, and the main function of given scheme has realized. The idea of design scheme supplied in the paper,which is also applied in other CAN distributed-control system, is that resultful combination the high-speed of MC9S12D64 microcontroller with the high reliability and real-time of CAN field-bus. The system was certified to the national Ministry of Communications, and has been applied successfully to the actual projects in some cities in China, and its results are satisfying.

\section{Introduction}

In recent years, performances of many integrated on-chip MCU systems are improved largely, at the same time, the relevant software and development tools are becoming perfect, with the price of MCU dropped. Microprocessor based on control, which integrated high-powered processor kernel and plenty of microcontroller peripherals, appeared, and rapidly became an ideal substitute for traditional multi-microprocessor unit. In traditional road traffic data-collecting management system, usually, a small quantity of processing operations about traffic data were dealt by several distributed processing units, its main problems are that system's operational stability and data-transmitting reliability is so poor, the ratio of performance and price (here its abbreviation is RPP) is not good. With the RPP and integrating degree of on-chip MCU being increased, the above existing problems were solved or were improved at a different degree already, so that, its more far-ranging application in ITS is foreseed.

\section{Design of System scheme}

\section{Introduction of 9S12D64 characteristic}

The MC9S12D64 microcontroller unit (MCU) is a 16-bit device composed of standard on-chip peripherals including a 16-bit central processing unit (HCS12 CPU), 64K bytes of Flash EEPROM, $4 \mathrm{~K}$ bytes of RAM, 1K bytes of EEPROM, two asynchronous serial communications interfaces(SCI), one serial peripheral interface(SPI), an 8-channel IC/OC enhanced capture timer, two 8-channel, 10-bit analog-to-digital converters (ADC), an 8-channel pulse-width modulator(PWM), a digital Byte Data Link Controller (BDLC), 29 discrete digital I/O channels (Port A, Port B, Port K and Port E), 20 discrete digital I/O lines with interrupt and wakeup capability, a CAN2.0 A, B software compatible modules (MSCAN12), and an Inter-IC Bus.

\section{System's whole structure design}

In traditional industry field control system communication often appears hardware structure, difficult to equip with a system of wire and to extend further. Supply in the paper CAN Bus acts as communication network to link each node in the whole system, each field intelligent measure equipment links with CAN Bus through the main controller. 9S12D64 is hard-core in the data-collecting system, in which includes some peripheral circuits, signal inductive equipment, high-speed and CAN transceiver (TJA1050T), provides CAN-Bus interface circuits, reequip with 
rs232 serial interface to communicate with the host. The main node in system structure is shown as Figure 1.

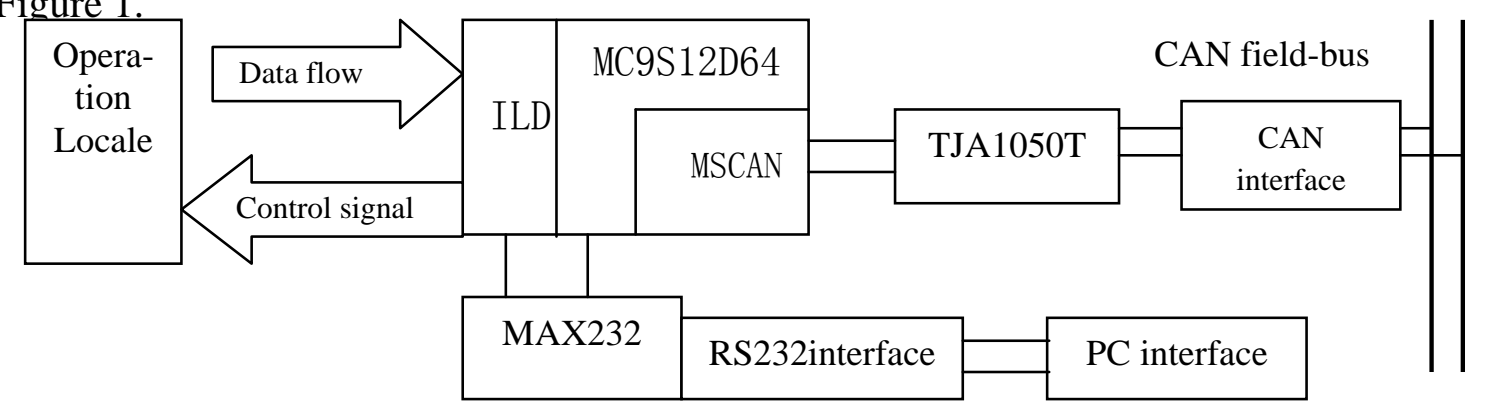

Figure 1 system structure master node sketch map

ILD in system includes signal optimized circuits and signal processing equipment. Under the control of 9S12D64 single-chip, Traffic data from 4 forward channels of ILD in the system can display then and there, and is saved in MSF---FLASH (in addition 512K) to be propitious to use or examine timely. Moreover, able to control field detecting equipment through ILD control module, able to transmit the processed data gathered to network by CAN interface --- transceiver, or through rs232 interface to host.

\section{Design of system software}

\section{Frequency detecting and data storage}

Frequency detecting is to make use of real-time pulse capture and counting function of 9S12D64 enhanced capture timer. Frequency value is a time difference calculated from the pulse numbers and free counter. The codes is written in compile language, and operated in interruption function.

Frequency storage here uses a defined buffer, that is, a two-dimension array structure, which can store 16 sampled frequency values from one of channels:

\section{Processing method for traffic data}

Suppose four inductive loops, which frequencies figure $f_{i}(t) \mathrm{i}=1,2,3,4$. Sensitive degree for inductive loops is supposed a percentage in advance, according to frequency the values needed to calculate as follows:

1) According to given sensitive degree, to distinguish whether a vehicle comes into inductive loops layed;

2) Enforce one dimension 5 dots Median filtering waves to frequency from inductive loops;

3) Carry out differential coefficient calculation to get differential time value.

Dynamic storage to sequential detecting frequencies from each inductive loop, supposed current time is letter $t$, so frequency values stored in Buffer are $f_{i}(t), f_{i}(t-1), f_{i}(t-15)$. As soon as a new sampled frequency arrives in Buffer, the oldest sampled frequency should be thrown away.

1) One dimension 5 dots Median filtering waves to sampled frequency

$$
\bar{f}_{i}(t)=\operatorname{middle}\left(f_{i}(t-2), f_{i}(t-1), f_{i}(t), f_{i}(t+1), f_{i}(t+2)\right)
$$

For the sake of convenience to express, after that, all the frequency value used to calculate is frequency value filtered as above.

2) Search inductive loop's no-vehicle frequency value $f_{i}(0)$

If the uninterrupted number of fulfilling the inequation $f_{i}(t)-f_{i}(t-15)<-60$ exceeds 10,it indicates frequency descending edge arrives. After that, if the uninterrupted number of fulfilling the inequation $\left|f_{i}(t)-f_{i}(t-15)\right|<10$ exceeds 375, the frequency average here is considered to the background frequency value $f_{i}(0)$.

3) Calculate differential coefficient value

$$
f_{i}^{\prime}(t)=\left(f_{i}(t)-f_{i}(t-15)\right)
$$

4) Calculate interval 
If the uninterrupted number of fulfilling $f_{i}(t)-f_{i}(0)>$ given threshold value reaches 3 ,it indicates vehicle goes into given inductive loops,note the time $T_{i}(1)$; when the uninterrupted number of fulfilling $\left|f_{i}(t)-f_{i}(0)\right|<$ given threshold value reaches 3, note the time Ti (2). In turn, the two time Ti+1 (1) and Ti+1 (2) from the second inductive loop arrive here. Supposed letter ${ }^{L_{i}}$ stands for the inductive loop's length here.

5) Calculate the length and mean speed of vehicle

Mean speed of vehicle $\mathrm{Vi}=540 * l_{i} /\left(\mathrm{Ti}+1(1)-T_{i}(1)\right)$, letter $l_{i}$ stands for center distance of the two inductive loops at the same roadway;

Length of vehicle $=(\operatorname{Ti}(2)-\mathrm{Ti}(1))^{*} l_{i} /\left(\mathrm{Ti}+1(1)-T_{i}(1)\right)-L_{i}$.

\section{System's development and realization}

Modularization design ideas are used in course of system's software development. System's software development mainly includes four sections: traffic data-collecting software, data-processing software, rs232 communication software and CAN Bus communication software.

\section{Software for traffic data-collecting}

Traffic data-collecting modularization is to make use of group of loop sensors distributed on roadway samples the real-time traffic data in the field, then to use median filtering waves to filters the sampled frequencies, and stores the processed frequencies into the given buffers, to prepare for the next data-processing modularization.

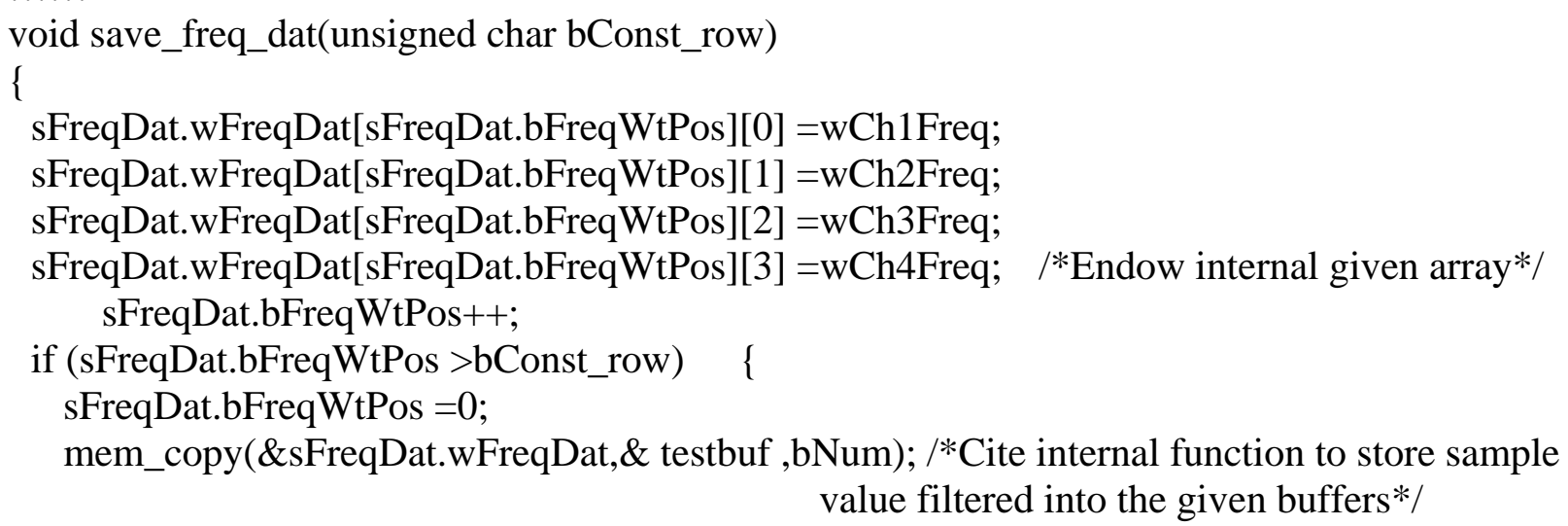

\section{Traffic data-processing software}

Traffic data-processing modularization is that using some control arithmetic to process the sampled frequencies stored in given buffers, here includes data filtering waves and signal compensation; users here need write some proper program codes on the basis of users' requirement and custom. Here give an example to calculate vehicle's speed and length, judge overspeed and overlength.

void dataprocess(void) \{

if(sCfgFile.wParafileExist!=1) return; /*Judge whether to download configuration parameters*/

Divdata

$$
=
$$


- (unsigned int)sCfgFile.bLoopLenght[0];

/*Calculate vehicle's speed and length*/ if(sVehicle.Road[0].wSpeed > sCfgFile.bUpSpeedLimit) ／* Judge whether to overspeed*/

$\{\quad \ldots . .$. \}

sVehicle.Roadstatus[0].mask_overspeed=1;

if(sVehicle.Road[1].wLength > sCfgFile.LargLengthUpLimit) /*Judge whether to overlength*/

$\{\ldots . .$. \}

sVehicle.Roadstatus[1].mask_overlength=1;

\}

\section{Communication}

Depend on rs232 interface and internal SCI module of 9S12D64 singlechip to realize rs232 communication. Rs232 interface module is protected with ESD and discharging diode. The section includes ports' initialization, refering to the receiving and sending function. Ports' initialization is to set the baud rate register, control register 1 and control register 2, which is carried out in whole system's initialization function. Here pay especial attention to match communication baud rate between serial communication interface and CAN Bus. In SCI interruption function finishes receiving and transmitting data. Writing the receiving and transmitting function, which is so simple, is on the basis of current requirement, so that the two functions are left out. For example:

interrupt void sci0_isr(void)\{

/*Judge SCI0 status, Cite

receiving / transmitting function*/

if (SCIOSR1_RDRF)

sci0_recv(); $\quad / *$ Quote user's receiving function*/

if (SCIOSR1_TC ||SCIOSR1_TDRE) function*/

sci0_tran(); $\quad / *$ Quote user's sending

\}

\section{System testing}

The system, so far as being, has examined by quality examination department belonging to the national Minister of communication, and its performance indexes answer the special demands completely. Its parameters examined mainly include: the vehicle's speed, the vehicle's length (the two in preceding text mentioned), the motorcade's length, the traffic flow and time occupancy ratio (algorithm is simple, no descriptions in article) etc. Looked from use result of the system in some city of Shandong province, by the end of March, 2006 the examination result: vehicle detecting error ratio less than $1 \%$; speed average error less than $3 \%$, at low speed to $1 \%$; traffic flow error less than $5 \%$, greater for some large vehicles or trailers to $15 \%$; vehicle length error less than $10 \%$; motorcade length error less than

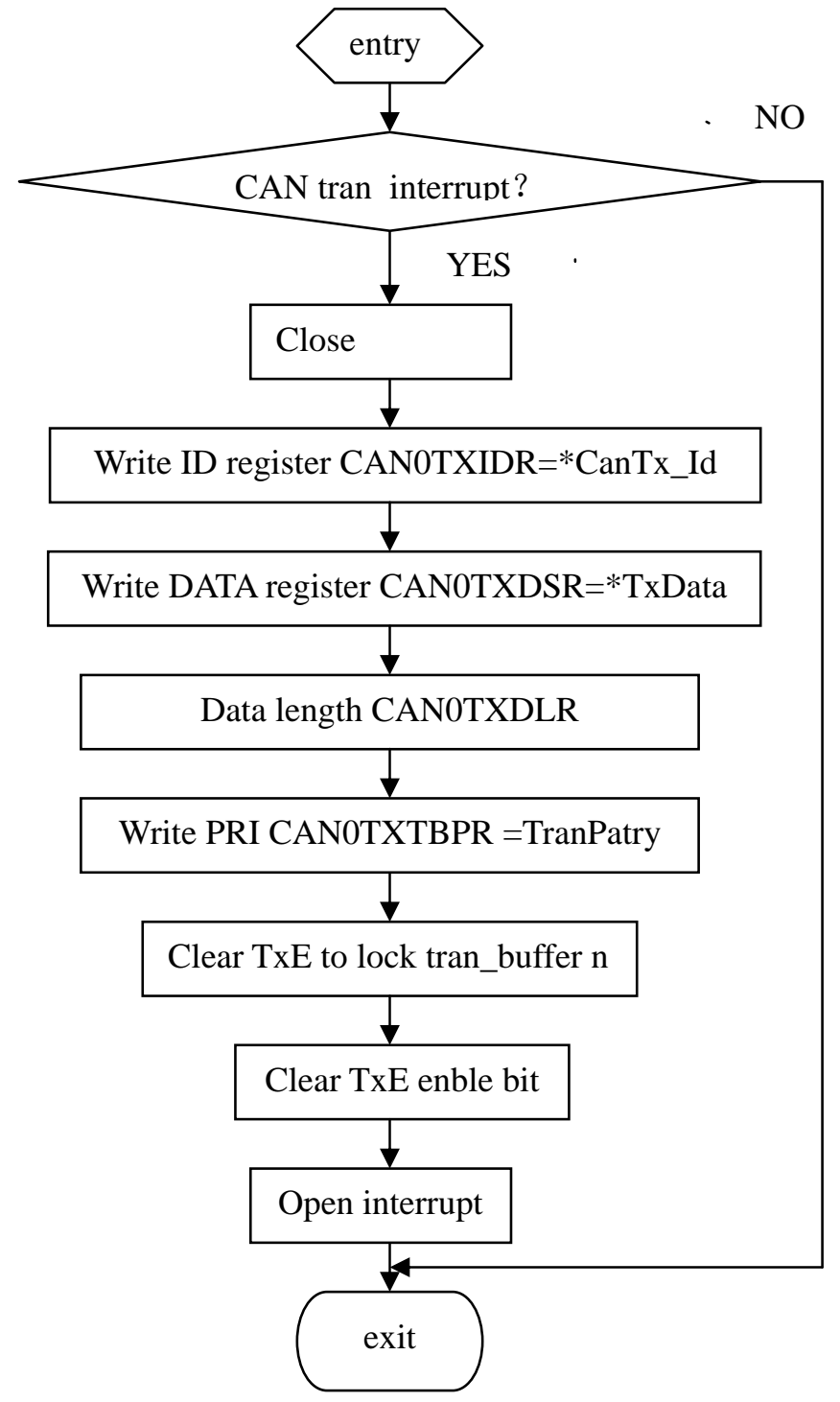

Figure 3 Transmission interrupt flow 
4 meters; time occupancy ratio error less than 5\%. Main parameters are showed in Table 1. (Note: Valid speed range $10-250 \mathrm{~km} / \mathrm{h}$, usual use range $20-130 \mathrm{~km} / \mathrm{h}$. The valid vehicle length scope $3-30 \mathrm{~m}$.)

Table 1 System testing result

\begin{tabular}{|l|c|c|c|c|c|c|}
\hline & $\begin{array}{l}\text { Average } \\
\text { speed }\end{array}$ & $\begin{array}{l}\text { Low } \\
\text { speed }\end{array}$ & $\begin{array}{l}\text { Average } \\
\text { length }\end{array}$ & $\begin{array}{l}\text { Vehicle } \\
\text { detecting }\end{array}$ & $\begin{array}{l}\text { Motorcade } \\
\text { length }\end{array}$ & $\begin{array}{l}\text { Time occupancy } \\
\text { ratio }\end{array}$ \\
\hline error & $<3 \%$ & $<2 \%$ & $<10 \%$ & $<1 \%$ & $<4 \mathrm{~m}$ & $<5 \%$ \\
\hline
\end{tabular}

Note: error $=\mid$ real value - detected value $\mid$ or $\mid$ real value - detected value $\mid * 100 \%$ / detected value.

In addition, connecting the large numbers of loop detecting cards (16 cards is testing number, that is 16 nodes) through CAN Bus, to form a local area network, namely, is a multi-cards traffic data-collecting system, which may satisfy the vehicle detecting, using multi-node network, in the multiple roadways road-traffic information gathering system in some large and middle scale cities, its parameter types examined, the precision or the error are equivalent with those of the single card system almost.

\section{Conclusions}

Supply in the paper a method to research and develop a road traffic data-collecting system centered 9S12D64 microcontroller, which RPP is so high, and provided with CAN, widely can use in the data-collecting projects for ITS, is that resultful combination the high-speed of MC9S12D64 microcontroller with the high reliability and real-time of CAN field-bus. The system not only can realize the traffic data centralism processing and the long-distance transmission, also can realize the on-line system's reconfiguration, which are convenient to realize the scene equipment plugging and playing and the modularization, distributed system structure transformation of data-collecting system. In the article introduce with emphases the key technique of system's software development and realization. The practices demonstrate that system's operation is stable and data transmission is credible, it can meet the practical project application completely. The design idea supplied in the paper, about road traffic data-collecting system, also meets the other situations requested the high reliability and high real-time, has the certain application value.

\section{References}

[1] Motorola, Motorola Semiconductor Technical Data, 2000

[2] TOSHIBA, TLP115A Technical Data sheet, 2002, 9

[3] Liu Zhiyong, Intelligent Transportation Control Theory and Application, Beijing: Science Press, 2003

[4] Computer and Communication,Editorial Department of House of Computer and Communications, 2004, (5)

[5] S. Liu, Z. Zhu. Design and Realization of Intelligent Measuring and Controlling Node Based on CAN Bus. Modern Electronics Technique. 2003, (3)

[6] Rao Yuntao, Field Bus CAN Principle and Application Technology, Beijing: Beihang Press, 2003

[7] B. Have, A. Kirby. Transparent Interconnection of Local Area Network with Bridge. Telecommunication Network. 1984, 3(2) m

[8] TJA1050 High-speed CAN Tranceiver, Guangzhou Zhouligong Singlechip Development Ltd.co, http://www.zlgmcu.com

[9] CAN basic knowledge, Guangzhou Zhouligong Singlechip Development Ltd.co, http://www.zlgmcu.co 